\title{
Secondary Procedures in Replantation
}

\author{
S. Raja Sabapathy, MS, MCh (Plastic Surgery), FRCS (Ed) ${ }^{1} \quad$ Praveen Bhardwaj, FNB (Hand \& Microsurgery) $^{1}$ \\ ${ }^{1}$ Department of Plastic Surgery, Hand Surgery, Reconstructive \\ Microsurgery \& Burns, Ganga Hospital, Coimbatore, Tamil Nadu, \\ India \\ Semin Plast Surg 2013;27:198-204. \\ Address for correspondence S. Raja Sabapathy, MS, MCh (Plastic \\ Surgery), Department of Plastic Surgery, Hand Surgery, \\ Reconstructive Microsurgery, Ganga Hospital, 313 Mettupalayam \\ Road, Coimbatore - 641 043, Tamil Nadu, India \\ (e-mail: rajahand@vsnl.com).
}

\begin{abstract}
Keywords

- replantation

- secondary surgery

- tenolysis

- hand amputation

The success of replantation surgery is not judged by survival of the replanted part, but by the functional outcome attained. Hence, primary repair of all injured structures is the preferred aim. At times, constraints induced by the ischemia time and nature of injury preclude primary repair. In such situations, secondary procedures are inevitable. Secondary procedures are also frequently required to improve the function and appearance of the replanted extremity. The incidence of secondary procedures will vary with the level of replantation and the type of patient population. Secondary procedures are difficult because they carry risk of injury to the vital neurovascular structures that now lay at nonanatomical locations. Nevertheless, when indicated and performed with caution they could significantly raise the functional status of the individuals.
\end{abstract}

Primary reconstruction of all injured structures is the preferred aim in hand injury management. It is more important to practice this principle in replantation surgery because secondary procedures following replantation are more difficult to do and carry some risk of injury to the anastomosed vessels. Some replants remain dependent upon the primary anastomosis for a long time and hence protection of the arterial anastomosis during secondary procedures is safer.

At times, due to the nature of injury and the constraints dictated by the ischemia time, full formal repair of all structures may not be possible. This makes secondary reconstruction inevitable. The incidence will vary with the level of replantation and the type of patient population. Wang ${ }^{1}$ found that the incidence of secondary procedures addressing this topic in published series ranged from 2.95 to $93.2 \%$. Yu et al ${ }^{2}$ in a retrospective study found $32 \%$ of their cases with replants and revascularization distal to the wrist required secondary operations. Major replants may need even more secondary operations. Yaffe et $\mathrm{al}^{3}$ in their series of 22 successful major replants found that six patients with arm replants required 17 operations and 16 cases of forearm replants required 50 secondary operations over 2 years.

We propose that secondary procedures in replantation surgery could be grouped under three headings.
1. Repair of structures that were not repaired during the primary procedure. This would mainly involve bridging gaps in tendons and nerves.

2. Procedures to promote healing or enhance function of tissues that were primarily repaired. This would involve surgeries like bony procedures for the correction of malunions and non-unions and tenolysis.

3. Procedures to enhance function like selective arthrodesis of joints, lengthening of bones, and tendon transfers to maximize the function of the recovered muscles.

\section{General Considerations}

\section{Documentation of the Original Operation}

Availability of good operation notes of the primary procedure can make the planning and execution of the secondary procedures less complicated. In a replant, the repaired vessels and nerves do not always lie in their regular anatomical location. Although it is possible to locate the position of the arteries by Doppler, there is no definite way to locate important venous anastomosis or the nerve repair routes. Although the site of the proximal end of the nerve can be identified by the Tinel sign, there is no easy way to preoperatively locate the distal end of the nerve. Knowing them will help us design safe access incisions. Documentation of the ends of the nerves
Issue Theme Replantation; Guest Editor, S. Raja Sabapathy, MS, MCh (Plastic Surgery), FRCS (Ed)
Copyright @ 2013 by Thieme Medical Publishers, Inc., 333 Seventh Avenue, New York, NY 10001, USA. Tel: +1(212) 584-4662.
DOI http://dx.doi.org/ 10.1055/s-0033-1360587. ISSN 1535-2188. 
and tendons will help to limit the access incisions and make the secondary procedures in replants much easier and safer.

\section{Timing and Sequence of Secondary Procedure}

The recommended timing for secondary procedures does not vastly differ between replantation patients and other hand injury patients. Though no fixed period can be provided for different secondary procedures, certain guidelines can be given. The important consideration is that there must be supple skin coverage in the area to be operated. Usually this stage is reached by 3 months. Infection, delayed healing of the wounds, failure to graft spotty raw areas after the primary procedure delay this process. When one anticipates that secondary procedures would be necessary, efforts must be taken to reduce edema and early healing of all wounds. Early healing will facilitate application of compression garments and institution of full-fledged physiotherapy. Mobilization programs help to reduce edema and induration around the operated sites.

Obtaining skeletal stability is the first requisite when planning secondary reconstruction. If nerve reconstruction is needed, we must be looking for an early opportunity to perform the nerve reconstruction. If tendon reconstruction is also required, it is preferable to do them together. It is better to complete the reconstruction of bone and tendons prior to or together with nerve reconstruction. This is because of the risk of injuring nerve grafts at the subsequent surgery.

\section{Nature of Soft Tissue Cover}

Good soft tissue cover is essential prior to secondary procedures following replantation. The line of access incisions and the passage of nerve and tendon grafts must have good soft tissue cover (-Fig. 1). Skin grafts might have to be replaced with flaps prior to tendon and nerve reconstruction. If a flap cover had previously been provided, the junction of the flap skin and the native skin must become supple before proceeding with tendon or nerve reconstruction. Adhesions of the tendon grafts are more common at the flap-native skin interface.

\section{Repair of Structures Not Repaired during the Primary Procedure}

\section{Secondary Reconstruction of Tendons}

Each area has peculiar technical characteristics and the issue is dealt with according to the different parts of the limb.

\section{Arm: Secondary Repair of the Elbow Flexors | Extensors}

In arm amputations, if the nature of injury is crush or avulsion in nature, the biceps get avulsed proximally and a significant amount of muscle hangs attached to the distal tendon. Brachial artery anastomosis will not revascularize the muscle attached to the distal tendon. All the muscle attached to the distal tendon needs to be excised during debridement. Arm replantation patients in whom the primary repair of biceps was not possible will present in two ways: not enough of proximal muscle available for use and significant proximal biceps available for repair.
In the first situation (which will more commonly be encountered), the best solution to restore elbow flexion is the transfer of functional pedicled latissimus dorsi (LD) muscle. Care is taken to mark the route of the vessels, and skin flaps are raised to enable the bipolar transfer of LD muscle to be done. Schoeller et $\mathrm{al}^{4}$ used this procedure in five arm replantations and at mean 43-month follow-up, three patients achieved M4 and two patients M3 elbow flexion power. Good results can consistently be achieved (-Fig. 2). This procedure has also been done in a primary sitting when it provides both flexion power as well as good soft tissue cover for the anastomosis sites. ${ }^{5}$

It can be done as both a unipolar and a bipolar transfer. ${ }^{6}$ When done as a bipolar transfer proximally the attachment is given to the coracoid process. To make the attachment secure, the latissimus dorsi muscle has to be harvested until its insertion into the humerus. Distally the attachment is more demanding because at times, the LD at the level of the distal attachment is muscular and it has to be weaved round the biceps tendon attachment into the radius. The elbow is immobilized in 90 degrees of flexion for 4 weeks. Then a detachable splint is applied for 4 weeks and gradual loading and strengthening exercises are started.

Sometimes the level of vessel anastomosis is in the proximal third of the arm or axilla and tunneling the pedicle of the LD flap from the posterior region of the trunk may be difficult. In such situations, Clark's pectoralis major transfer can be used. ${ }^{3}$ The pectoralis muscle has to be harvested with a strip of the rectus abdominis fascia. This will help to obtain good distal attachment. This transfer has the advantage that the whole operation can be done in the supine position, but leaves the individual with a scar in the front of the chest.

If a significant amount of innervated proximal biceps muscle is available, fascia lata may be used to connect the muscle and the distal insertion of the biceps tendon. Secure healing at both ends is essential and the technique practiced by the authors is useful (-Fig. 3). A 1-inch fascia lata strip of adequate length is harvested and is split into two until the last 2 inches. The unsplit portion is hooked around the biceps insertion and multiple horizontal mattress sutures of \#1.0 Prolene are used to securely suture the distal attachment. Keeping the elbow flexed to 110 degrees, the two strips of the fascia lata are weaved into the muscle, which has been under stretch for at least 5 minutes to overcome the shortening of the muscle fibers and to stretch them to its maximum limits. The weaved portion of the tendon is anchored by \#3.0 Prolene sutures (-Fig. 3). Triceps muscle, due to its flatter insertion and widespread origin, does not often get separated out in avulsion injuries like the biceps. With the bone shortening that is regularly done during replantation, it might be possible on most occasions to repair the triceps. Pedicled latissimus dorsi flap could also be used as a functioning muscle transfer for the reconstruction of triceps. When both are lost, reconstruction of biceps takes priority.

\section{Reconstruction at the Forearm Level}

Reconstruction of gaps in the tendons in the forearm is a challenge if one end is muscle and the other ends are tendons. 
A
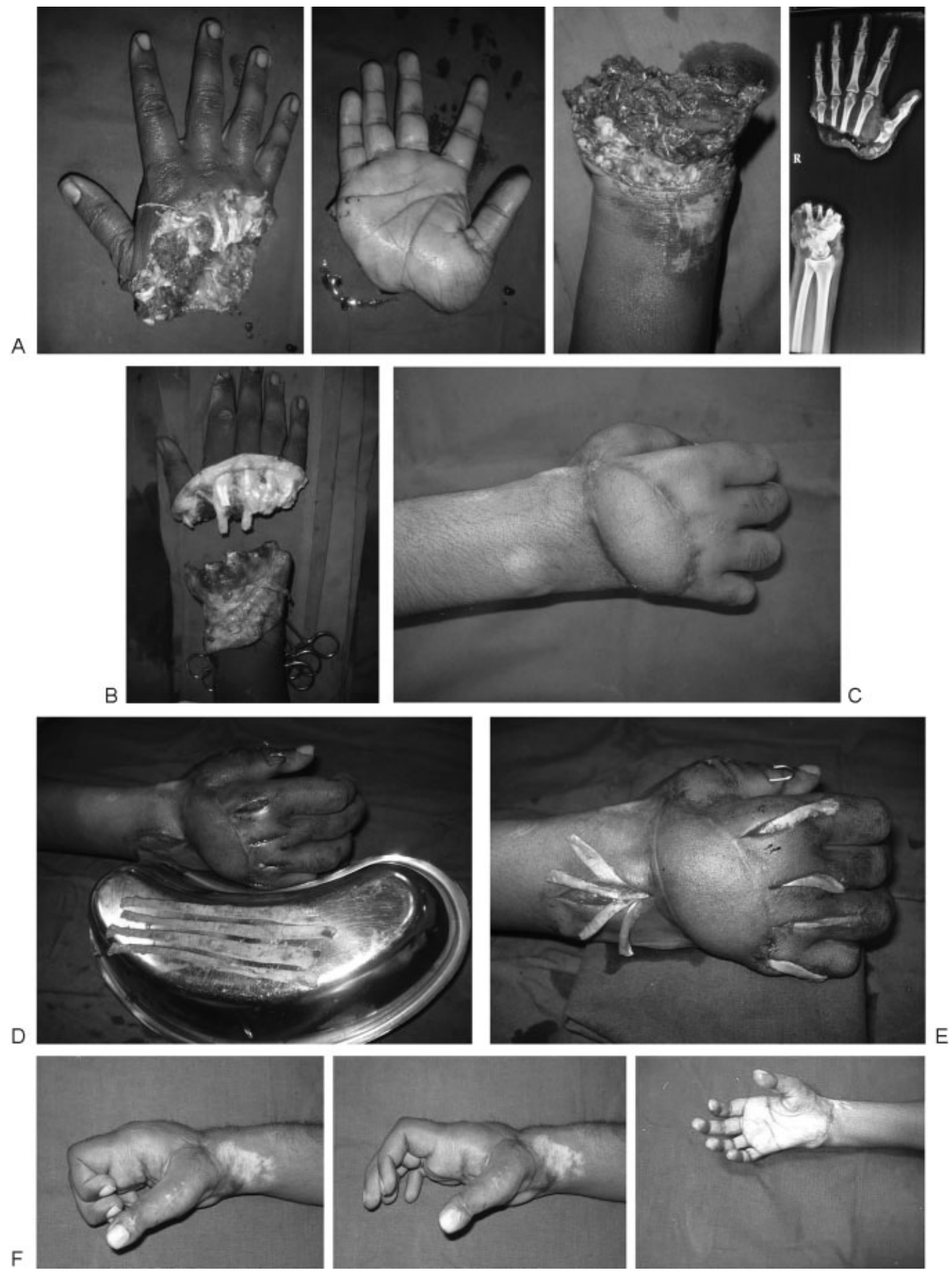

$E$
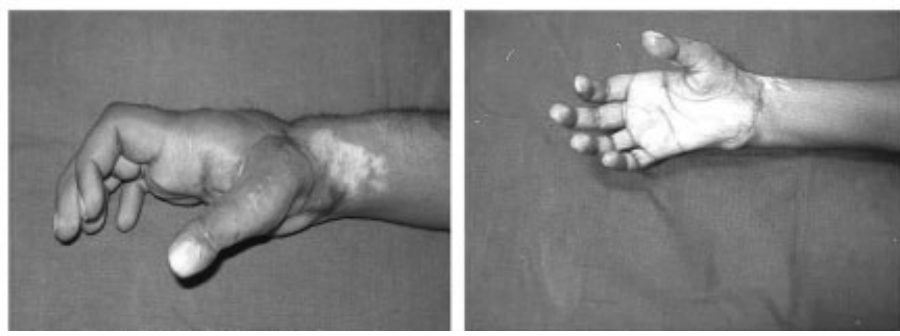

Fig. 1 (A) A 24-year-old man who presented with crush avulsion amputation at the trans-metacarpal level. (B) Postdebridement picture of the hand. There was segmental loss of extensor tendons over the zone VI. (C) The skin over the dorsum of the hand had partial necrosis that required an abdomen flap at a later stage. Pliable skin is a must to allow future extensor tendon reconstruction. (D) Extensor tendon cut ends exposed proximal to the extensor retinaculum proximally and proximal to the metacarpophalangeal joint distally. (E) The gap bridged by strips of fascia lata grafts. The flap at the dorsum allowed easy passage of the tendon grafts underneath.(F) Function of the same hand 1 year postsurgery showing satisfactory opening up of the fingers for grasping and good flexion of the fingers.

Attachment of the distal tendons to proximal muscles directly or through fascia lata grafts produces some flexion power. This is functionally useful to produce a stabilizing effect when the hand is used as an assisting hand to the other hand.
Reconstruction at this level has to be individualized by studying the musculotendinous units present on both sides. When the amputation results in avulsion within the muscles at or proximal to the neuromuscular junction with the entire 
A
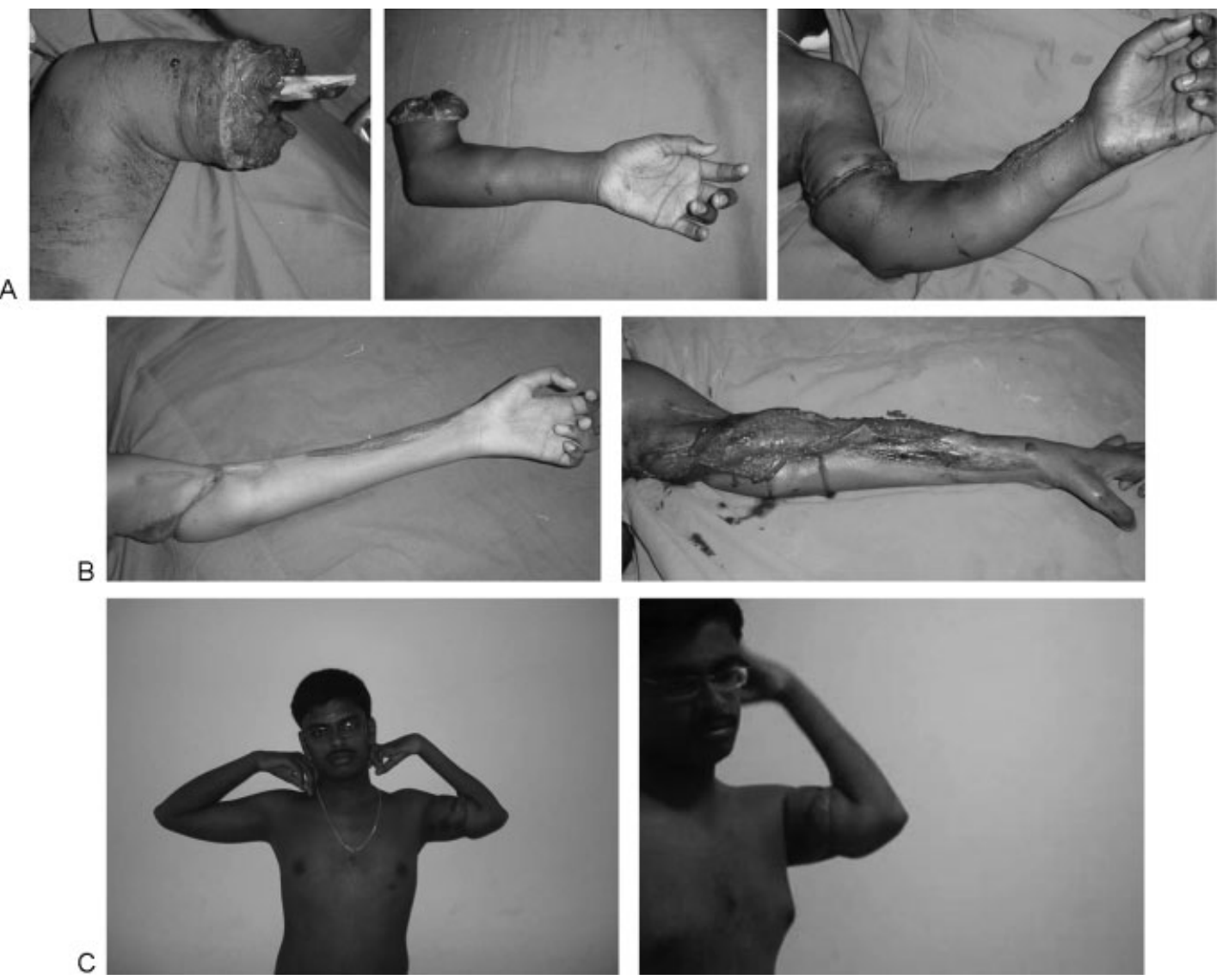

Fig. 2 (A) A 14-year-old boy presented with avulsion amputation at the arm level, which was successfully replanted. There was a segmental crushing of biceps muscles precluding its primary repair. (B) One year postreplantation the reconstruction of elbow flexion done using pedicled latissimus dorsi muscle flap. (C) Six years postreplantation pictures: patient has grade IV elbow flexion and good overall function of the limb.

muscle being denervated or destroyed (Chuang type 3 injury), ${ }^{7}$ a free functioning muscle transfer is useful to gain function.

\section{Reconstruction in the Hand}

Primary repair of tendons may not be possible in avulsion injuries. Frequently, the problem is in the approximation of the extensors after the flexors have been repaired. It has been shown that reasonable amount of hand function is possible without extensor function and that flexor rehabilitation is more important. ${ }^{8}$ In secondary reconstruction of extensors, care has to be taken not to limit flexion as a result of extensor tightness during reconstruction. On no account should extensor reconstruction limit the flexion range of movement or the ability to make a fist.

On rare occasions, primary flexor tendon repair is not possible. Placement of silicone rods and later use of tendon grafts for flexor reconstruction has been recommended. ${ }^{9}$

\section{Secondary Reconstruction of the Nerves}

In replantation of a major avulsion amputation of the upper limb, it might not be possible to suture the nerves. It is not advisable to make extra incisions or prolong the operation time during the primary sitting to trace the proximal nerve ends of the nerves if they are not easily available. In such situations, we recommend placing the distal nerve end in an easily accessible location. If a long distal segment is available, it could be drawn proximally by a tendon tunneler in the anatomical line of the nerve and placed in a subcutaneous position. Its position is marked on the skin by a scratch mark (-Fig. 4). At a later date, by making a small incision, the distal end can be identified and freshened for formal repair. The proximal end can be located by the presence of the Tinel sign. The proximal nerve segment could be mobilized and often direct repair might become possible ( - Fig. 4 ). If there is a nerve gap, the grafts could be tunneled between the two incisions. This will make the procedure easy and avoid the risk of injuring vital structures.

When planning secondary reconstruction, attempts are made to repair both median and ulnar nerves. If only one proximal nerve is available, then grafts could be shared to connect both the median and ulnar nerves. Initially, priority was given to median nerve reconstruction so that it could provide sensation to the contact surface of the hand. Our experience with major replants has made us think that ulnar nerve repair is also equally important and has the potential to provide useful function. This is due to the possibility of intrinsic muscle recovery. Recovery of the adductor of the thumb in itself could make the hand functional by providing a side key pinch between the ulnar aspect of the thumb and the 

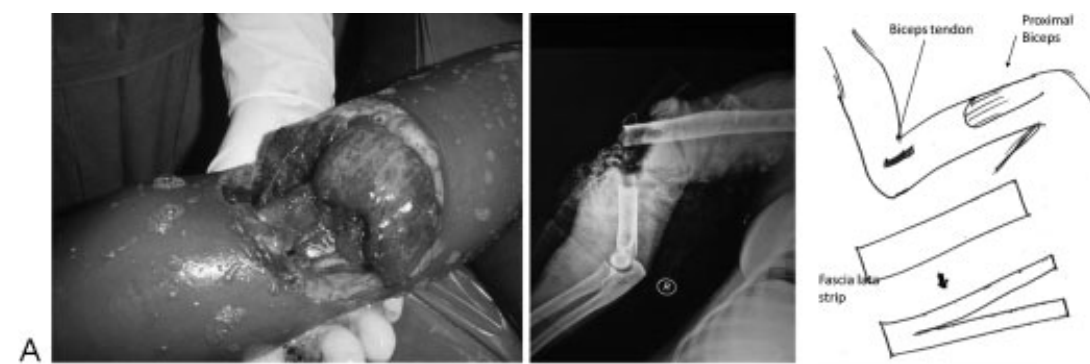

B
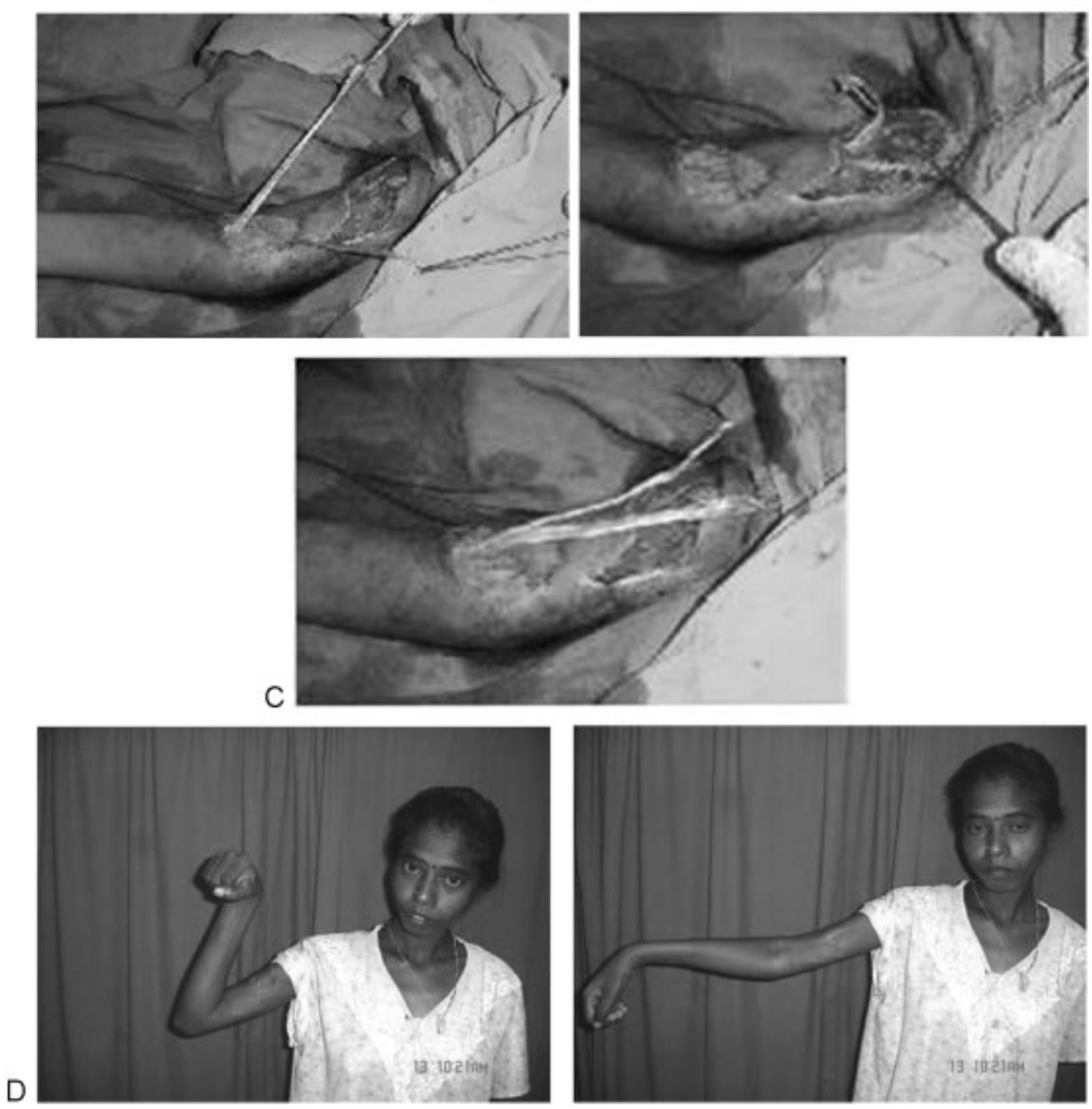

Fig. 3 (A) A 23-year-old woman who presented with near total amputation at the arm level with segmental loss of biceps muscle. She underwent successful revascularization. At second stage 8 months postinjury biceps reconstruction was planned. (B) Line diagram showing the scheme of biceps reconstruction using fascia lata as a bridging graft. We split the fascia lata into two except for the one end. This broad end is sutured circumferentially around the distal biceps tendon and two divided strips are individually and separately weaved into the biceps muscle mass proximally. (C) Intraoperative photographs showing the fascia lata being sutured to the biceps tendon distally and muscle mass proximally. (D) One year postsurgery photographs showing good elbow flexion and full extension. Patient had grade IV elbow flexion.

index finger. Over time, the patient learns to capitalize this single movement and use it for many activities. ${ }^{10}$

Nerve transfers are new options in secondary reconstruction after replantation. It is useful to either motor a useful muscle or provide sensation in the contact surface of the fingers.

\section{Procedures to Promote Healing or Enhance Function of the Repaired Structures}

Stable skeletal stabilization is an important step in replantation surgery. It allows early mobilization of the tendon repairs. Sometimes, due to the speed needed in replantation surgery or due to the nature of the fractures, bony fixation may not be ideal. It could result in non-union. The incidence of non-union could be as high as $10 \%$ in digital replantations. ${ }^{11}$ Bone shortening, which is an integral part of replantation, helps reduce the incidence of non-union. Sabapathy et $\mathrm{al}^{12}$ in their report on 20 successful major replantations found that bone shortening was achieved during primary surgery by proximal row carpectomy in three cases and the average bone shortening in the forearm and arm was $7.4 \mathrm{~cm}$ (range 5-10 $\mathrm{cm}$ ). With such extensive shortening, they did not have a single case of non-union. ${ }^{12}$ Established non-unions need to be treated in the usual way of bone grafting. Bone union has to be achieved before performing other secondary procedures.

Tenolysis is the commonest secondary procedure that might be needed in the hand. Care has to be exercised not to injure the vessels and nerves that have been repaired in the first sitting. In replantation surgery, the metacarpophalangeal 
A

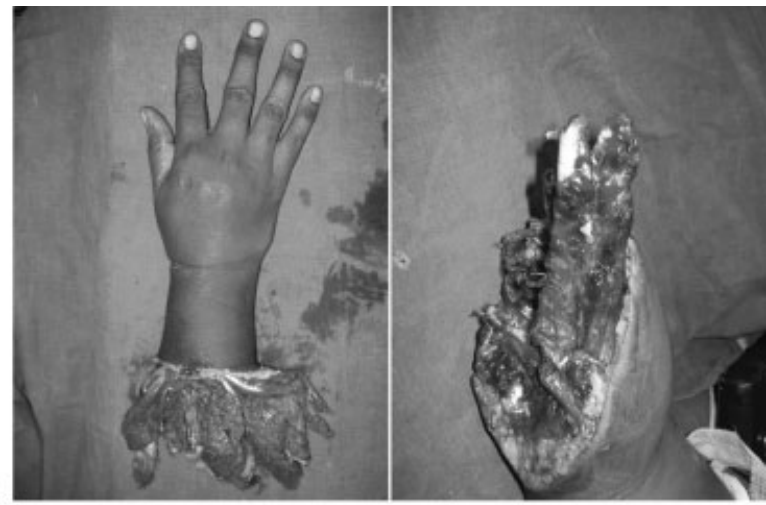

B
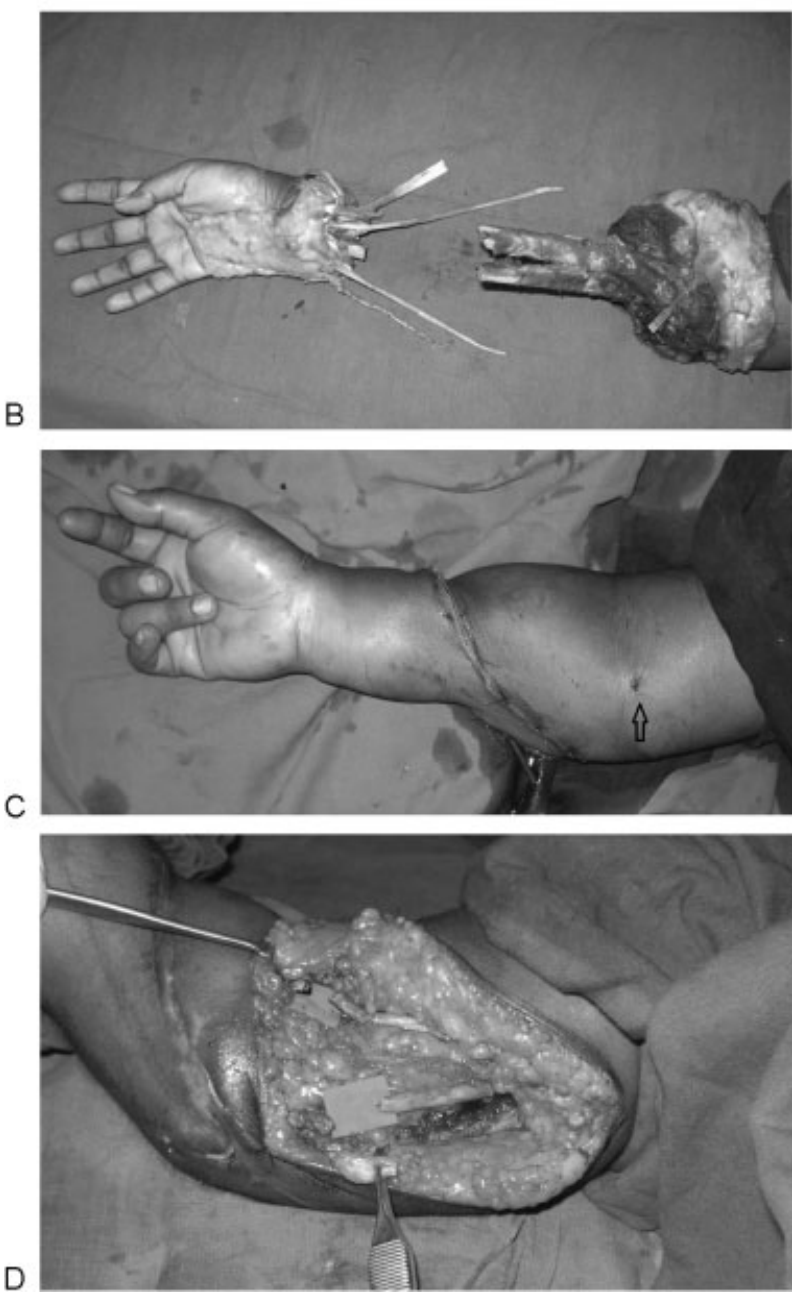

Fig. 4 (A) A 24-year-old woman presented with a machinery crush avulsion amputation at the midforearm level. (B) Postdebridement picture showing the avulsed median nerves. Such avulsed nerves may not be possible to suture primarily and it is not advisable to make extra incision proximally to explore the proximal cut end and prolong the operation. (C) The forearm was successfully replanted. The avulsed nerves were retrieved proximally using a tendon tunneler and banked subcutaneously at easily accessible location that would not risk the vital structures during secondary exploration (arrow). The location can be determined by the scar mark. (D) Four months postreplantation excess incision made at the site of previous scar mark over the banked nerves and nerves could be repaired end to end (shortening during replantation allowed end-to-end repair of the nerves). and interphalangeal joints may also become stiff and simultaneously require attention. Gaining flexion is more important than gaining extension; often flexion is limited by extensor tetherance. If extensors have not been repaired, the distal end gets adherent and it is a common cause of limitation of flexion. Release of it allows flexion to occur.

Flexor tenolysis is indicated when the passive range of flexion at the finger is more than the active range and the progress has plateaued during rehabilitation. The timing of tenolysis is not defined, but any time after 4 months should be safe. In the study by Jupiter et al, ${ }^{13}$ the average time of flexor tenolysis was 10 months after replantation (range 5-19 months). Because tenolysis should follow early postoperative mobilization, it should be performed only after all the procedures requiring immobilization are over and the overlying soft tissue is supple. If the procedure is done under local anesthesia and intravenous sedation, the surgeon can be certain of the extent of the release by asking the patient to move the fingers after tenolysis. If the procedure is done under general anesthesia or brachial block, the "traction flexor check" recommended by Whitaker et $\mathrm{al}^{14}$ is useful. The involved tendon is exposed through a separate incision at the wrist and pulled to estimate the potential range of motion. This also helps in breaking the adhesions that have been missed during tenolysis.

The choice of incision may be at times dictated by the previous surgery scars. Long zig-zag incisions are preferred as they allow the exposure of the entire length of the tendon, are easy to extend, and can incorporate the previous scars. The entire length of the flexor tendon in the region of expected adhesions is exposed and the adhesions are methodically excised. It is imperative to preserve the critical A2 and A4 pulleys. If the preservation of the pulleys is not possible, one can opt to reconstruct the pulleys at the time of tenolysis or resort to two-stage tendon reconstruction. At times, the quality of the tendon at the repair site is very poor because of rupture and gap formation resulting in tendon rupture. In this situation, tendon grafting or tendon transfer should be contemplated. The tenolysis must follow a dedicated mobilization protocol.

Good results have been reported with flexor tenolysis after replantation. Jupiter et al ${ }^{13}$ reported their results in replantation of 37 fingers and four thumbs. They noted an increase of total active motion from a mean pretenolysis of 72 degrees to 130 degrees, which was a statistically significant improvement $(p<0.001)$. Their results were not encouraging when the procedure was done for the thumb. ${ }^{13}$ Poor results were noted in crush or avulsion amputations, hands with more than two digits amputated, and those requiring proximal interphalangeal joint capsulotomy. Yu et $\mathrm{al}^{2}$ reported increase in total active motion from 119 degrees pretenolysis to 159 degrees. Only one of their 21 patients had deterioration of total active motion after tenolysis.

\section{Procedures to Enhance Function}

In major crush injuries, tendon transfers may frequently be offered to increase the functional level of activity. But in 
replantation, very rarely tendon transfers are done. Alternatively, selective arthrodesis of joints are done to provide stability or to change the movement arc.

Finger joints are commonly arthrodesed joints. In some major replantations, long flexors and extensors recover without significant intrinsic recovery. In such cases, the interphalangeal joints flex first and roll into the palm. The thumb in the absence of intrinsics cannot oppose properly and there is no thumb-fingertip pinch movement. The long flexors have good flexion power, but only hook function is possible. In such situations, arthrodesis of the proximal interphalangeal joint in $\sim 10$ degrees flexion transfers the flexion movement to the metacarpophalangeal joint. It makes the fingertips meet the thumb better and pinch grip becomes possible. Metacarpophalangeal joint and the interphalangeal joints of the thumb are the joints that may be stabilized for enhancing function. A trial splint is given for a week in the proposed position of arthrodesis before performing the definitive procedure.

\section{Lengthening of the Replanted Part}

Bone shortening is a routine procedure in replantation surgery to remove the traumatized and nonviable tissues, to allow end-to-end repair of the neurovascular structures without need of grafting, and to achieve adequate soft tissue cover. In digits, a shortening of $1 \mathrm{~cm}$ and in the forearm $\sim 3$ to $5 \mathrm{~cm}$ is accepted functionally and aesthetically. Sometimes in critical replants like replantation of the crush-avulsion amputation of the thumb or distal forearm replants with segmental crushing of tissues excessive shortening is needed, which may be a functional and/or cosmetic problem. In such cases, the replanted part can be lengthened using distraction osteogenesis principles.

Unal et $\mathrm{al}^{15}$ reported a case of lengthening of a thumb distal phalanx replanted to its metacarpal because of loss of the proximal phalanx. There was a shortening of $4 \mathrm{~cm}$ in their case and they performed $2.5-\mathrm{cm}$ lengthening using unilateral minidistraction device at 6 months postreplantation. The distraction was started on the $10^{\text {th }}$ day after surgery at interval of $1 \mathrm{~mm} / \mathrm{d}$ until a length of $25 \mathrm{~mm}$ was achieved. The fixator was kept in place for 4 months to allow ossification of the callus and no bone grafting was required. At the time of removal of fixator the first web was deepened. They reported good functional and cosmetic outcome in their case, and the patient was satisfied with the result. Vucetic ${ }^{16}$ reported a case of forearm elongation after hand replantation in a patient who required $13-\mathrm{cm}$ shortening during replantation because of segmental tissue damage. At 6 months postreplantation, they used Ilizarov apparatus to lengthen the forearm by $7 \mathrm{~cm}$ and also to correct the ulnar deviation and flexion deformity of the wrist. Their patient was extremely satisfied with the cosmetic and functional outcome. ${ }^{16}$

\section{Conclusion}

Secondary procedures following replantations are difficult to do and primary repair of all structures is advisable. Nevertheless, when indicated and performed with due precautions they could significantly raise the functional status of the individuals. Choosing the optimal timing of intervention, limiting the length of access incisions and taking care to avoid injury to the critical structures followed by supervised physiotherapy are the key to achieving success.

\section{References}

1 Wang $\mathrm{H}$. Secondary surgery after digit replantation: its incidence and sequence. Microsurgery 2002;22(2):57-61

2 Yu JC, Shieh SJ, Lee JW, Hsu HY, Chiu HY. Secondary procedures following digital replantation and revascularisation. Br J Plast Surg 2003;56(2):125-128

3 Yaffe B, Hutt D, Yaniv Y, Engel J. Major upper extremity replantations. J Hand Microsurg 2009;1(2):63-67

4 Schoeller T, Wechselberger G, Hussl H, Huemer GM. Functional transposition of the latissimus dorsi muscle for biceps reconstruction after upper arm replantation. J Plast Reconstr Aesthet Surg 2007;60(7):755-759

5 Haas F, Hubmer M, Koch H, Scharnagl E. Immediate functional transfer of the latissimus dorsi myocutaneous island flap for reestablishment of elbow flexion in upper arm replantation: two clinical cases. J Trauma 2004;57(6):1347-1350

6 Kawamura K, Yajima H, Tomita Y, Kobata Y, Shigematsu K, Takakura Y. Restoration of elbow function with pedicled latissimus dorsi myocutaneous flap transfer. J Shoulder Elbow Surg 2007; 16(1):84-90

7 Chuang DC, Lai JB, Cheng SL, Jain V, Lin CH, Chen HC. Traction avulsion amputation of the major upper limb: a proposed new classification, guidelines for acute management, and strategies for secondary reconstruction. Plast Reconstr Surg 2001;108(6):1624-1638

8 Quaba AA, Elliot D, Sommerlad BC. Long term hand function without long finger extensors: a clinical study. J Hand Surg $[\mathrm{Br}]$ 1988;13(1):66-71

9 Beris AE, Darlis NA, Korompilias AV, Vekris MD, Mitsionis GI, Soucacos PN. Two-stage flexor tendon reconstruction in zone II using a silicone rod and a pedicled intrasynovial graft. J Hand Surg Am 2003;28(4):652-660

10 Sabapathy SR, Venkatramani H, Bharathi RR, Bhardwaj P. Replantation surgery. J Hand Surg Am 2011;36(6):1104-1110

11 Pomerance J, Truppa K, Bilos ZJ, Vender MI, Ruder JR, Sagerman SD. Replantation and revascularization of the digits in a community microsurgical practice. J Reconstr Microsurg 1997;13(3):163-170

12 Sabapathy SR, Venkatramani H, Bharathi RR, Dheenadhayalan J, Bhat VR, Rajasekaran S. Technical considerations and functional outcome of 22 major replantations (The BSSH Douglas Lamb Lecture, 2005). J Hand Surg Eur Vol 2007;32(5):488-501

13 Jupiter JB, Pess GM, Bour CJ. Results of flexor tendon tenolysis after replantation in the hand. J Hand Surg Am 1989;14(1):35-44

14 Whitaker JH, Strickland JW, Ellis RK. The role of flexor tenolysis in the palm and digits. J Hand Surg Am 1977;2(6):462-470

15 Ünal MB, Cansü E, Parmaksizoğlu F. Lengthening of a thumb distal phalanx replanted to its metacarpus because of loss of the proximal phalanx: case report. J Hand Surg Am 2011;36(4):661-664

16 Vucetic CS. Forearm elongation after hand replantation. A case report. J Bone Joint Surg Am 2005;87(1):181-186 